\title{
Prothyroliberin Release
}

National Cancer Institute

\section{Source}

National Cancer Institute. Prothyroliberin Release. NCI Thesaurus. Code C40615.

Any process in which the tripeptide hormone prothyroliberin is released from the hypothalamus, processed and bound to receptors in the anterior pituitary, resulting in sustained release of prolactin from the anterior pituitary. Thyroliberin may be elevated in primary hypothyroidism, resulting in elevated prolactin secretion. This process is involved in regulating the levels of both prolactin and thyroid-stimulating hormone. 